Supporting Information for

\title{
A semi-stable emulsion system based on spent cooking oil for pilot-scale reactive dyeing with minimal discharges
}

Linyun Liu ${ }^{\text {a }}$, Bingnan $\mathrm{Mu}^{\mathrm{b}}$, Wei Li ${ }^{\mathrm{a}, \mathrm{b}, *}$ and Yiqi Yang ${ }^{\mathrm{b}, \mathrm{c}, \mathrm{d}, *}$

${ }^{\text {a }}$ Key Laboratory of Science \& Technology of Eco-Textiles, Ministry of Education, Jiangnan

University, 1800 Lihu Road, Wuxi, Jiangsu 214122, China

${ }^{\mathrm{b}}$ Department of Textiles, Merchandising and Fashion Design, 234, HECO Building, University

of Nebraska-Lincoln, Lincoln, NE 68583-0802, United States

${ }^{\mathrm{c}}$ Department of Biological Systems Engineering, 234, HECO Building, University of Nebraska-

Lincoln, Lincoln, NE 68583-0802, United States

${ }^{\mathrm{d}}$ Nebraska Center for Materials and Nanoscience, 234, HECO Building, University of Nebraska-

Lincoln, Lincoln, NE 68583-0802, United States

* Corresponding Author: Yiqi Yang, E-mail: yyang2@unl.edu

* Co-corresponding Author: Wei Li, E-mail: vivianlee305@ huskers.unl.edu

The Supporting Information file has 9 pages, including 5 equations (Eq. S1, Eq. S2, Eq. S3, Eq.

S4 and Eq. S5) and 4 tables (Table S1, Table S2, Table S3 and Table S4) and 1 figure (Figure S1). 


\section{Langmuir isotherm model}

The Langmuir isotherm model assumes that the dyes forms a monolayer coverage on a homogeneous surface of the dyeing substance. Linearized equation of the Langmuir isotherm model is expressed as Eq. S1:

$$
\frac{[D]_{s}}{[D]_{f}}=\frac{[D]_{s}}{[S]_{f}}+\frac{1}{K_{L}[S]_{f}}
$$

In which $[D]_{s}$ is the concentration of RR23 in the internal phase at equilibrium $\left(\mathrm{g} \mathrm{L}^{-1}\right) ;[D]_{f}$ is the concentration of RR23 on cotton fabrics at equilibrium ( $\left.\mathrm{mg} \mathrm{g}^{-1}\right) ;[S]_{f}$ is the saturated concentration of RR23 on cotton fabrics $\left(\mathrm{mg} \mathrm{g}^{-1}\right) ; K_{L}$ is the Langmuir constant that relates to the affinity between RR23 and fabrics $\left(\mathrm{L} \mathrm{mg}^{-1}\right)$.

\section{Freundlich isotherm model}

The Freundlich isotherm model is an empirical formula assumes that multilayer coverage of dyes occurred on a heterogeneous surfaces and non-uniform distribution of adsorption heat over the surface without a saturation of adsorption sites. Linearized equation of Freundlich isotherm model is given as Eq. S2:

$$
\ln [D]_{f}=\ln K_{F}+\frac{1}{n} \ln [D]_{s}
$$

In which $[D]_{f}$ is the concentration of RR23 on cotton fabrics at equilibrium $\left(\mathrm{mg} \mathrm{g}^{-1}\right) ;[D]_{s}$ is the concentration of RR23 in the internal phase at equilibrium $\left(\mathrm{g} \mathrm{L}^{-1}\right) ; K_{F}$ is the Freundlich constant; $\mathrm{n}$ is the heterogeneity factor.

Affinity between dyes and fabrics was calculated based on Eq. S3.

$$
-\Delta \mu^{o}=R T \ln K_{L}[S]_{f}
$$


In which $R$ is the ideal gas constant $\left(1.9872 \mathrm{cal} \mathrm{mol}^{-1} \mathrm{~K}^{-1}\right) ; T$ is the temperature $(\mathrm{K}) ; K_{L}$ is the Langmuir constant that relates to the affinity between RR23 and fabrics $\left(\mathrm{L} \mathrm{mg}^{-1}\right) ;[S]_{f}$ is the saturated concentration of RR23 on cotton fabrics $\left(\mathrm{mg} \mathrm{g}^{-1}\right)$.

\section{Pseudo-first-order and pseudo-second-order models}

The pseudo-first-order model assumes that the rate change of dye-uptake with time is directly related to the difference in saturation concentrations, while the pseudo-second-order model assumes that the adsorption rate determining step may be a chemical surface reaction.

The pseudo-first-order model follows Eq. S4:

$$
\ln \left([D]_{f}-[D]_{t}\right)=\ln [D]_{f}-k_{1} t
$$

The pseudo-second-order model follows Eq. S5:

$$
\frac{t}{[D]_{t}}=\frac{1}{k_{2}[D]_{f}^{2}}+\frac{t}{[D]_{f}}
$$

In which $k_{1}\left(\min ^{-1}\right)$ and $k_{2}\left(\mathrm{mg} \mathrm{g}^{-1} \mathrm{~min}^{-1}\right)$ are rate constants of pseudo-first-order and pseudosecond-order kinetics equations, respectively. $[D]_{t}\left(\mathrm{mg} \mathrm{g}^{-1}\right)$ is the concentration of RR23 on cotton fabrics at time $t,[D]_{f}$ is the concentration of RR23 on cotton fabrics at equilibrium (mg $\left.\mathrm{g}^{-1}\right)$

\section{Mobile phase for HPLC analysis}

A mixture of acetonitrile and isopropanol were used as the mobile phase. In 15 min, percent of acetonitrile decreased from $80 \%$ to $40 \%$, while percent of isopropanol increased from $20 \%$ to $60 \%$. Flow-rates were $1.0 \mathrm{~mL} \mathrm{~min}^{-1}$ at $30{ }^{\circ} \mathrm{C}$. 
Table S1. Mobile phase used in HPLC analysis to determine dye reactivity with glycerol and water.

\begin{tabular}{ccc}
\hline Time (min) & Acetonitrile (\%) & Isopropanol (\%) \\
\hline 0 & 80 & 20 \\
15 & 40 & 60 \\
\hline
\end{tabular}

\section{Statistical analysis of $\mathbf{9 5 \%}$ confidence interval of Langmuir fittings}

By analyzing the $95 \%$ confidence interval of Langmuir fittings for the conventional aqueous dyeing system and the semi-stable emulsion dyeing system, it could be concluded that there is no significant difference in fixation behaviors of dyes between the two systems ( $\mathrm{P}$ value $=0.7749$ ), as shown in Table S2.

Table S2. Differences between the 95\% confidence interval of Langmuir fittings for the two dyeing systems.

\section{Estimates}

\begin{tabular}{ccccccc}
\hline Label & Estimate & $\begin{array}{c}\text { Standard } \\
\text { Error }\end{array}$ & DF & t Value & Pr $>|\mathbf{t}|$ & Alpha \\
Fitting & & & & & & \\
difference & 0.000195 & 0.000668 & 13 & 0.29 & 0.7749 & 0.05 \\
\hline
\end{tabular}

Swelling of cotton in oil, water and the emulsion under dyeing conditions

The swelling behavior of pre-conditioned cotton in oil, water and the emulsion under dyeing conditions is shown in Table S3. Though cotton fibers treated by spent cooking oil have their 
average diameter "smaller" than that of untreated cotton fibers, such two types of fibers are statistically the same in their diameters, indicating little swelling. Such result is consistent with the negligible number of solvent molecules per anhydroglucose unit in cotton fabrics treated by oil. Oil molecules could enter the large voids in cotton fibers; however, they are difficult to break strong hydrogen bonding between cellulose to form small voids in fibers. Such small voids other than the large voids are the major contributor of substantial increase (about 40-70 times) in the specific surface area of cellulose. Without the formation of the small voids, the swelling of cotton in oil becomes negligible.

The slight difference in average diameters of cotton fibers treated by oil and untreated fibers is due to the unique nature of cotton fibers. A cotton fiber looks like a twisted ribbon, with its fineness varied at different positions. The convolutions also give cotton fibers an uneven surface. Therefore, experimental error could be large, leading to the slight difference (not necessarily a decrease or an increase) in average diameter of cotton fibers after being treated by oil. The difference in swelling behaviors of cotton fibers in oil and water results in the difference in measurement errors of fiber diameters. Unlike oil, water remarkably swells cotton fibers, leading to a much more regular structure and thus, smaller measurement errors.

Cotton fabrics treated by the emulsion has a larger percentage of weight gain than those treated by pure water since the oil content could not be completely removed via centrifugation. The water content in the emulsion endows cotton fabrics with a percentage of weight gain of $30.98 \pm 0.32 \%$, which is not statistically different from that of cotton fabrics treated by pure water. In addition, 
pre-conditioned cotton fabrics treated by the emulsion and pure water do not have any statistically significant difference in their fiber diameters. Such results indicate that water other than oil swells the pre-conditioned cotton. Pre-conditioned cotton fabrics have the same swelling behavior in the emulsion dyeing system and the conventional aqueous system. Therefore, the two dyeing systems also have the same fixation behaviors of dyes, as demonstrated in Figure 4 (a) in the manuscript. Table S3. Swelling behavior of cotton in oil, water and the emulsion under dyeing conditions

\begin{tabular}{|c|c|c|c|}
\hline Solvent & $\mathrm{Sw} \%^{\dagger}$ & $\mathbf{n S w}^{\S}$ & Fiber diameter $(\mu \mathrm{m})$ \\
\hline Untreated & - & - & $6.82 \pm 1.90^{f}$ \\
\hline Oil & $14.84 \pm 0.17^{\mathrm{a}}$ & $0.03 \pm 0.00^{\mathrm{d}}$ & $6.01 \pm 1.52^{f}$ \\
\hline Water & $31.42 \pm 0.29^{b}$ & $2.83 \pm 0.03^{\mathrm{e}}$ & $14.70 \pm 0.55^{\mathrm{g}}$ \\
\hline Emulsion" & $45.82 \pm 0.57^{\mathrm{c}}$ & - & \\
\hline $\begin{array}{l}\text { Water content in fabrics } \\
\text { treated by the emulsion* }\end{array}$ & $30.98 \pm 0.32^{b}$ & $2.79 \pm 0.03^{\mathrm{e}}$ & $14.49 \pm 0.45^{\mathrm{g}}$ \\
\hline
\end{tabular}

$\dagger$ Swelling behavior is measured by percentage of weight gain.

$\S$ Swelling behavior is measured by the number of solvent molecules per anhydroglucose unit. The molecular weight of soybean oil $(900 \mathrm{~g} / \mathrm{mol})$ is used to estimate the $\mathrm{nSw}$ for cotton fabrics treated by spent cooking oil. The $\mathrm{nSw}$ for cotton fabrics treated by the emulsion is not included in the table since the fabrics contain both oil and water contents.

If Swelling behavior is quantified based on both water and oil contents in cotton fabrics treated by the emulsion. 
* Swelling behavior is quantified based on the water content in cotton fabrics treated by the emulsion.

a-g indicate statistically significant differences in data points.

\section{Effects of concentration of hydrolysates of oil on depth of shade}


Figure S1. Effects of content of glycerol and sodium fatty acid on dye fixation

Generation of the two hydrolysates of oil, glycerol and sodium fatty acids, both greatly decreased the dye fixation. Glycerol entered the internal phase during the dyeing process and 
competed with cotton to react with dyes. Fatty acids consumed alkali in the system. As a result, alkalinity in the internal phase was not high enough to fix all dyes onto fabrics.

\section{Comparison of material costs}

Table S4. Material costs of the conventional aqueous system and semi-stable emulsion system to dye $1 \mathrm{~kg}$ of cotton fabrics.

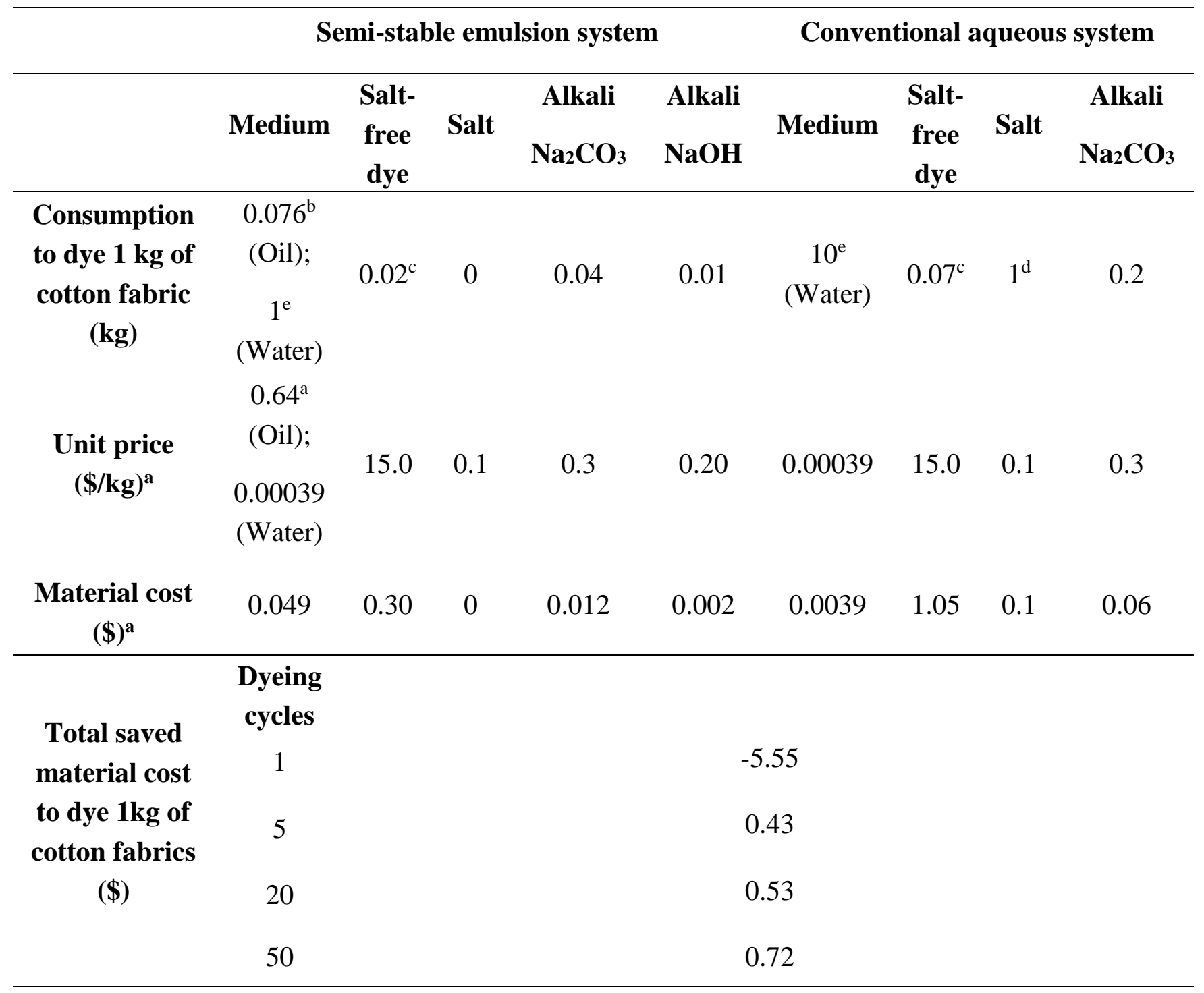


${ }^{a}$ All the prices of chemicals were obtained from Alibaba.com, accessed on 02/06/2019. The unit price of spent cooking oil is calculated based on a yield of $65 \%$ and includes the cost of alkali refining.

${ }^{\mathrm{b}}$ Consumption of oil: $1 \%$ lost during 3 cycles of dyeing due to hydrolysis; $0.07 \%$ lost during oil removal process after each cycle of dyeing.

${ }^{c}$ Dyed cotton fabrics are supposed to achieve a K/S value of 10 with the two dyeing systems. Thus, $2 \%$ owf and $7 \%$ owf of initial inputs of dyes are required for the semi-stable emulsion system and conventional aqueous system, respectively, based on our experimental results.

${ }^{\mathrm{d}}$ Sodium sulfate is added to the dye bath at $100 \mathrm{~g} \mathrm{~L}^{-1}$.

${ }^{\mathrm{e}}$ In the semi-stable emulsion system, water content has the same weight to fabrics. In the conventional aqueous dyeing, the fabric-to-water weight ratio is 1:10. 